\title{
Impact of Lower-extremity Strength on Shoulder Tackle of Female Rugby Players Measured with Sensor System
}

\author{
Xiaoneng Song, ${ }^{1,2} \mathrm{Hui} \mathrm{Xu},{ }^{1}$ Jie Meng, ${ }^{1}$ and Yigang $\mathrm{Wu}^{1 *}$ \\ ${ }^{1}$ School of Physical Education and Training, Shanghai University of Sport, \\ 650 Qing Yuan Huan Rd., Shanghai 200438, China \\ ${ }^{2}$ Sport Department, Jiangnan University, 1800 Li Hu Rd., Wu Xi, Jiangsu 214122, China \\ (Received January 11, 2021; accepted March 16, 2021; online published March 22, 2021)
}

Keywords: rugby, shoulder tackle, impact strength, force sensor, isokinetic strength

The purpose of this study was to understand the impact of lower-extremity strength on the shoulder tackle ability of Chinese elite female rugby players. The impact strength of a tackle and the lower-extremity strength were measured by force sensors and an isokinetic strength tester. The results provide a theoretical reference for the specialized strength training of shoulder tackle techniques, relevant technical learning, and sports injury prevention, as well as for improving the defensive ability of shoulder tackles. The lower-extremity isokinetic strengths of the hip, knee, and ankle and the shoulder tackle impact force of 18 Chinese elite female rugby players were measured at fixed angular velocities of 60 and 180\% by an IsoMed 2000 strength testing instrument and an impact force tester. SPSS 25 software was used to test for a normal distribution by single-sample Kolmogorov-Smirnov $(\mathrm{K}-\mathrm{S})$ and independent-sample t-tests and the Pearson correlation test. The following findings were obtained. (1) The bilateral lowerextremity hip, knee, and ankle flexors and extensors had an extremely significant difference $(P<0.01)$ at fixed angular velocities of 60 and $180 \%$, and the test results of the extensors were greater than those of the flexors. (2) The right knee and left ankle extensors of the lowerextremity isokinetic strength were related to the shoulder tackle impact force. On the basis of these findings, it is suggested that (1) the lower-extremity isokinetic strengths of the front knee and back ankle in dominant-side tackles should be used as indexes to evaluate shoulder tackle ability, and (2) the balanced development of lower-extremity strength can help athletes enhance the defensive capacity of shoulder tackles, promote technical learning, improve the stability of actions, and prevent sports injuries.

\section{Introduction}

In China, rugby is an emerging sport compared with other traditional sports and its foundations are still weak. The number of currently registered professional rugby players is only 1521 (897 male players and 624 female players). To improve the standing of rugby sports in China, China aims to use the participation of the women's national rugby union team in the Tokyo Olympic Games as an opportunity to promote rugby. Simultaneously, the assistance of

*Corresponding author: e-mail: Wuyigan08@sus.edu.cn https://doi.org/10.18494/SAM.2021.3265 
science and technology, as well as the cross-boundary and cross-type selection of athletes, will accelerate the expansion of seven-a-side rugby. In brief, the development potential of rugby is enormous in China.

The gap between the Chinese team and the top teams in the world is mainly manifested in the defensive ability of the shoulder tackle technique. According to statistics based on the videos of international rugby games, the points scored against the Chinese team in the women's rugby sevens final at the 28th Jakarta Asian Games were primarily due to the failure of shoulder tackles used for defense. In the 2018-2019 World Rugby Women's Sevens Series (USA), the rugby games between the Chinese team and the USA, England, and New Zealand teams were also characterized by high failure rates of shoulder tackles of 45,55 , and $80 \%$, far exceeding those of the opponents of 30,20 , and $8 \%$, respectively. This comparison directly revealed that a major current technical shortcoming of the women's national team is the weak defensive ability of shoulder tackles. At present, the national team urgently needs research results on improving defensive techniques, especially those on shoulder tackle actions, to facilitate sports training, solve practical problems, and enhance the defensive ability and overall competitiveness of the team.

There are a number of studies on the relationship between the lower-extremity strength and the shoulder tackle technique. Speranza et al. have recently found that in semi-professional rugby league games, the lower-extremity maximal and explosive strengths of male players are related to their proficiency in the shoulder tackle technique. ${ }^{(1-3)}$ Gabbett et al. measured the factors influencing proficiency in the shoulder tackle technique in male players and found that the correlation coefficient for lower-extremity strength was $9 \%$ in a multiple regression model. ${ }^{(4)}$ The lower-extremity strength has different defensive effects on shoulder tackles with different postures and is related to game results. ${ }^{(5)}$ In particular, the technical actions and strength of legs primarily affect shoulder tackle actions. ${ }^{(6)}$ The success of the shoulder tackle technique is correlated to the lower-extremity strength; the correlation coefficient between the shoulder tackle ability and the squat strength was found to be $0.77 .^{(3)}$ The correlation between the vertical squat jump performance and the shoulder tackle ability of athletes is also significant. ${ }^{(6-8)}$ In addition, the peak torque, relative peak torque, and flexor/extensor ratio of three lower-extremity joints were found to be related to the squat performance..$^{(9,10)}$

The relative peak torque and relative average power of the slow and fast isokinetic motion of lower-extremity joints reflect the maximum force and explosive force of the lower-extremity flexor and extensor muscles, respectively, and the structure and muscle force of lower-extremity joints maintain the balance and stability of shoulder tackles. In research on the lower-extremity strength of rugby players, Comfort et al. studied the peak torques and peak torque ratios of hamstrings and quadriceps during concentric and eccentric isokinetic knee movements at a fixed angular velocity of $60 \%$ s. They reported that the hamstring/quadriceps ratios were not significantly different between forwards and backs, as well as between the dominant and nondominant legs. In addition, flexor/extensor peak torque ratios are correlated with sports injuries. ${ }^{(11)}$ Brown et al. found that rugby league and rugby union players had different hip and knee strengths: the hip extensors of rugby union players and the knee flexors of rugby league players were weaker. This imbalance could directly affect the performance of rugby players in sprinting, 
changing direction, and kicking. ${ }^{(12)}$ Brown et al. reported that the hip and knee peak torques and flexor/extensor peak torque ratios were different between the dominant and non-dominant legs of college rugby players. The flexor/extensor peak torque ratios for college players were lower than those for professional athletes, and the hip and knee peak torques of forwards were greater than those of backs. ${ }^{(13)}$ Dobbs et al. also found that the ratios for non-dominant legs were greater than those for dominant legs, and recommended the use of the flexor/extensor peak torque ratio of the knee joint as an indicator to assess the preseason competitive state of players and to predict the potential for sports injuries. ${ }^{(14)}$ Anastasi and Hamzeh experimentally tested the isokinetic strength of the hamstrings of healthy female rugby union players and found that training of the hamstrings has great potential for improving the imbalance of bilateral hamstring strength and improving the dynamic athletic ability of lower limbs. ${ }^{(15)}$

Chinese experts Lu et al. measured the isokinetic strength of men's and women's national rugby union players attending the 2014 Incheon Asian Games. ${ }^{(16)}$ They found that the lowerextremity flexor/extensor ratios for Chinese female rugby players were higher than those for male rugby players. Moreover, the overall physical conditions of male and female rugby players in China were far behind the players in top rugby-playing countries, and their overall strength and systemic ability were also significantly weaker than those of the players of top teams. The research concluded that the low coordination between the truncus and the hip joint led to poor capacities in both offense and defense. In particular, the weak defensive ability of male and female athletes was a shortcoming of the specialized athletic abilities of Chinese rugby players and the main reason for their low competitiveness at the international level. ${ }^{(16)}$

Researches have carried out a series of explorations on the biomechanical characteristics of rugby players and built a solid theoretical foundation for the development of rugby sports. However, the research on the relationship between the biomechanical characteristics of elite female rugby players and the technical and tactical development of rugby sports is still insufficient. In particular, supplementary evidence is required for the correlation between the lower-extremity strength and the defensive effect of shoulder tackles. In this study, we selected Chinese elite female rugby players as the subjects to implement objective measurements and a characteristic analysis on their isokinetic hip, knee, and ankle strengths and to explore the impact of lower-extremity strength on shoulder tackle techniques. Our results provide a theoretical reference for the specialized strength training, technical learning, and sports injury prevention of Chinese rugby players and provide evidence for relevant research on rugby sports as part of their promotion in China.

\section{Methods}

\subsection{Subjects}

The 18 voluntary participants were composed of five players preparing for the 2020 Olympic Games, four China Youth Team members, four former China National Team members, and five members of a Shanghai team. Among them, 13 athletes were master level and five athletes were national first-grade sportswomen, with training experience from three to nine years. The 
participating players were required to have had no injury within the previous three months or to have had more than three months of continuous training since recovery (based on the time recorded in medical rehabilitation certificates). The test was arranged in the middle of a long training cycle and in the strength training unit of a short training cycle. The test was carried out over one week, with adjustments and arrangements made for special situations. The basic characteristics of the subjects are shown in Table 1.

\subsection{Experimental design}

The isokinetic strength testing instrument was an IsoMed 2000 isokinetic dynamometry system. The testing contents included peak torque, average power, flexor/extensor peak torque ratio, relative peak torque, and relative average power of the bilateral hip, knee, and ankle joints.

The testing scheme was designed to test the flexion/extension actions of the hip, knee, and ankle joints. In the preparation phase, the test requirements were explained to the athletes, which was followed by a 15 min warm-up comprising $10 \mathrm{~min}$ of treadmill running and $5 \mathrm{~min}$ of dynamic stretching. Furthermore, the athletes were given at least three familiarization trials for each joint and each speed until they performed the movement satisfactorily.

As a result of the shoulder tackle action being in the sagittal plane, the angular velocity of the ankle movement is less than $180 \%$ s (according to the experimental test results of a doctoral project). On the basis of this result and other research findings, in the formal testing, each joint was tested at fixed angular velocities of 60 and $180 \%$ s through concentric actions. At each velocity, five flexion actions and five extension actions were tested every time, and two to four test results were taken. There was a 3 min rest between the tests of contralateral homonymous joints, with a 40 min interval between tests on heteronymous joints. The methodology was strictly implemented by professional operators in accordance with the operating instructions, and the testing reports were printed automatically by the IsoMed 2000 system. The average of the four repetitions was used as the final value, with the parameters of the isokinetic strength test shown in Fig. 1 and Table 2.

The impact strength of shoulder tackles was tested by our self-developed strength testing system (Invention Patent Number: ZL201910594285.9), which consisted of hardware and software parts. The hardware part mainly included FlexiForce A502 sensors, a USB data acquisition card, and a computer to collect, convert, transmit, store, and display signals. The LabVIEW software part involved an application program to implement the design and control of the data acquisition program, as well as a hardware driver to set the working mode of the data acquisition card, as shown in Figs. 2 and 3.

The test was implemented indoors at a constant temperature of $22{ }^{\circ} \mathrm{C}$. After the $15 \mathrm{~min}$ warm-up, the habitual position of a shoulder tackle in competitions was adopted as the ready

Table 1

Participant characteristics.

\begin{tabular}{lcccc}
\hline Total $(N=18)$ & Age $(\mathrm{y})$ & Training experience $(\mathrm{y})$ & Height $(\mathrm{cm})$ & Mass $(\mathrm{kg})$ \\
\hline Mean \pm SD & $19.7 \pm 3.1$ & $5.8 \pm 2.2$ & $168.4 \pm 3.5$ & $62.9 \pm 5.3$ \\
\hline
\end{tabular}




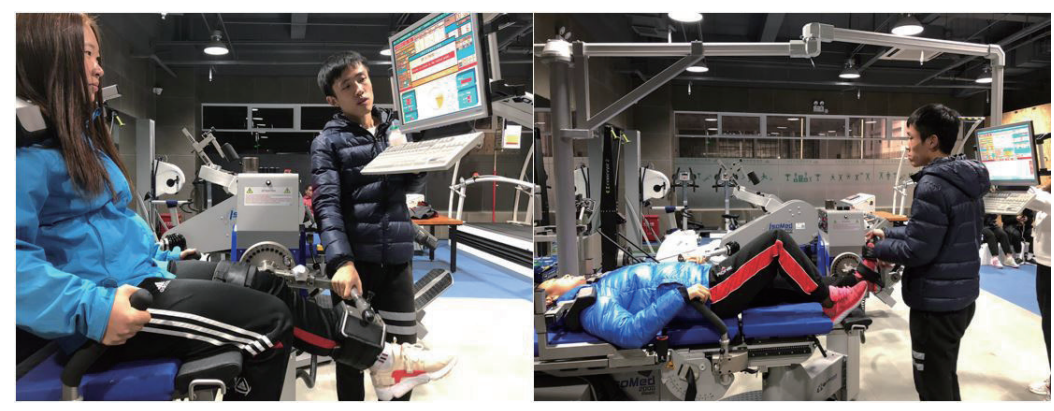

Fig. 1. (Color online) IsoMed 2000 isokinetic strength test.

Table 2

Isokinetic strength test parameters.

\begin{tabular}{lcclcc}
\hline Joint & Sports pattern & Action & \multicolumn{2}{l}{ Velocity $(\%)$} & Range of joint motion $\left(^{\circ}\right)$ \\
\hline Hip joint & Concentric & Flexion and extension & 60 & 180 & $-55-50$ \\
Knee joint & Concentric & Flexion and extension & 60 & 180 & $10-90$ \\
Ankle joint & Concentric & Flexion and extension & 60 & 180 & $-20-35$ \\
\hline
\end{tabular}
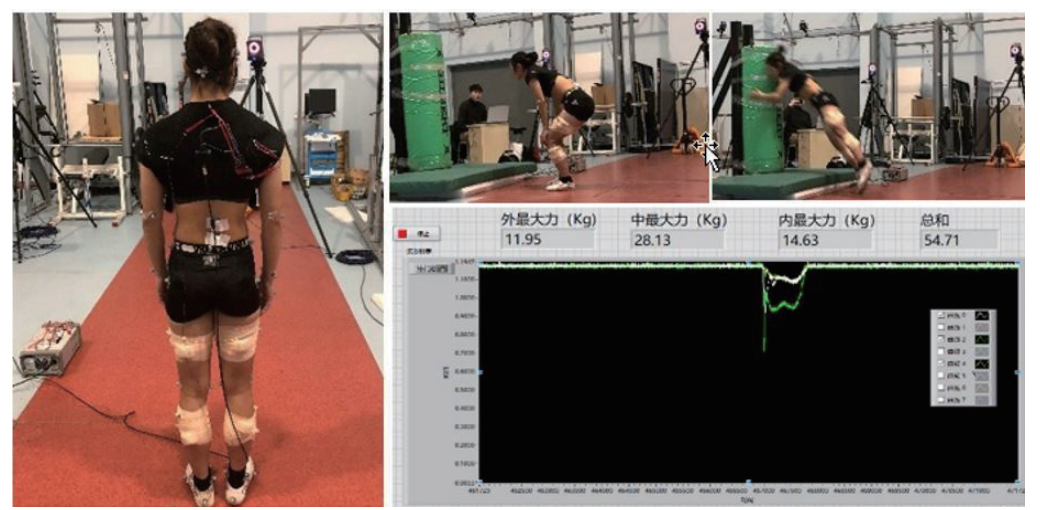

Fig. 2. (Color online) Application of impact strength testing system.

position. Starting from the static state on the force plate, the dominant shoulder in front and reverse shoulder tackles was used to perform three fully effective impacts, one each in the high, medium, and low positions. The time interval between each impact was $120 \mathrm{~s}$ and the average of the measurement results was taken for statistical analysis.

A parametric test was performed in SPSS (Version 25.0 for Windows), with a single-sample Kolmogorov-Smirnov (K-S) test performed to determine whether the testing data followed a normal distribution. For the data conforming to the parametric test, $Z$-score standardization was implemented to carry out divergence analysis, and the data exceeding the normalized value $(x-\mu / \delta),[-3,3]$ were removed. The statistical data of the isokinetic strength test were expressed as mean $\pm \mathrm{SD}$ and were examined through an independent-sample t-test, in which $P$ $<0.05$ was considered to be statistically significant and $P<0.01$ to be extremely significant. The correlation between the isokinetic strength of the lower-extremity joint movement and the impact strength of the shoulder tackle was examined through Pearson correlation analysis. 


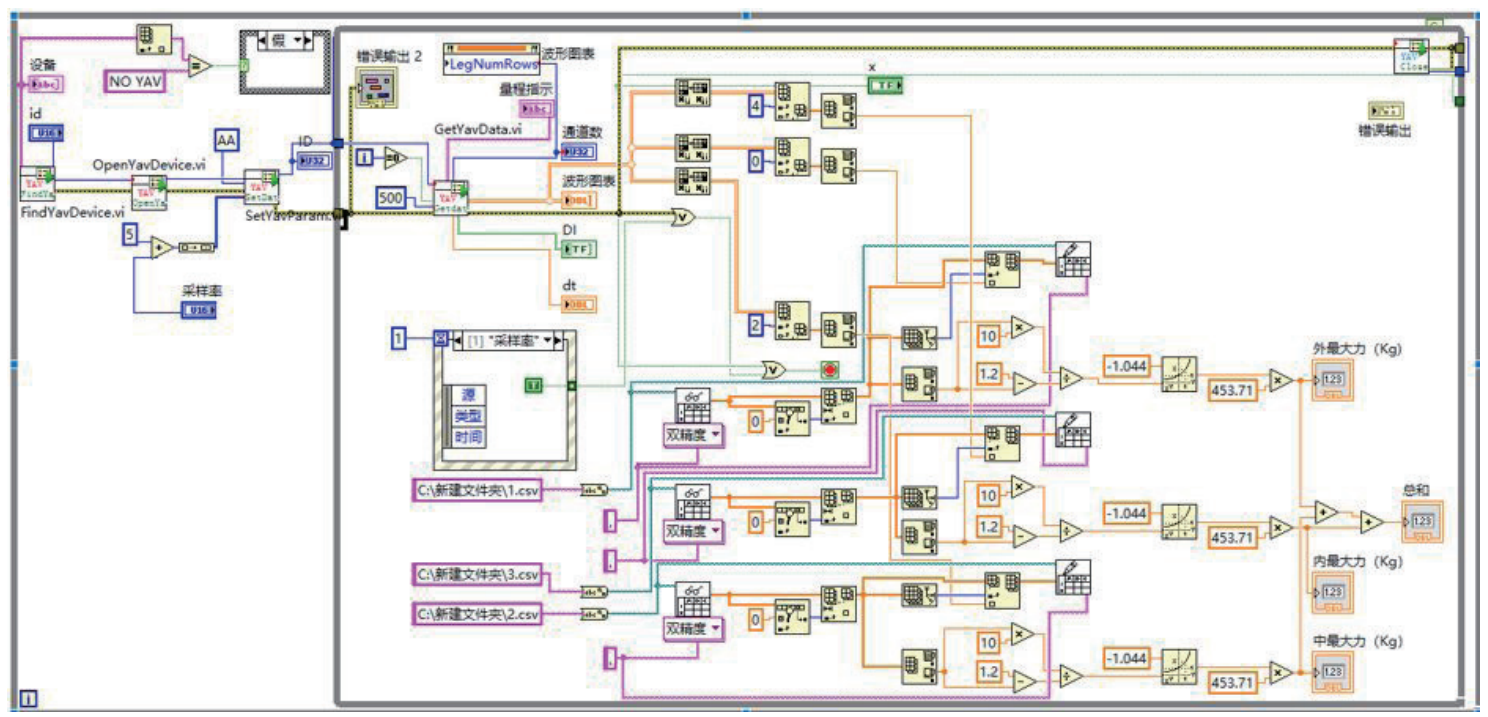

Fig. 3. (Color online) Program for acquiring data on shoulder tackle impact strength.

\section{Results}

\subsection{Peak torque and relative peak torque}

When the hip, knee, and ankle joints of the lower extremities moved at fixed angular velocities of 60 and $180 \%$, the peak torques and relative peak torques of the ipsilateral extensors were higher than those of the flexors, and their differences were extremely significant $(P<0.01)$. The detailed results are shown in Figs. 4 and 5.

\subsection{Correlation between lower-extremity isokinetic strength and impact strength of shoulder tackle}

The $\mathrm{K}-\mathrm{S}$ test was used to measure whether the data of the lower-extremity isokinetic strength and the impact strength of shoulder tackles followed a normal distribution. The results showed that $P>0.05$, indicating that the data followed a normal distribution and could be used in the Pearson correlation test, the results of which are shown in Table 3.

According to the analysis of the statistical data, at the fixed angular velocity of $60 \%$, the correlation between the relative peak torque of the left ankle extensors and three positions of the shoulder tackle was significant at a $95 \%$ confidence level. The three positions were the middle position of the front shoulder tackle, the low position of the front shoulder tackle, and the middle position of the reverse shoulder tackle. The relative peak torques of the right knee extensors and the low and middle positions of the front shoulder tackle had significant correlations at a $99 \%$ confidence level. The correlations between these relative peak torques and the high position of the front shoulder tackle, the high position of the reverse shoulder tackle, and the middle position of the reverse shoulder tackle were significant at the $95 \%$ confidence level, as shown in Table 4. 


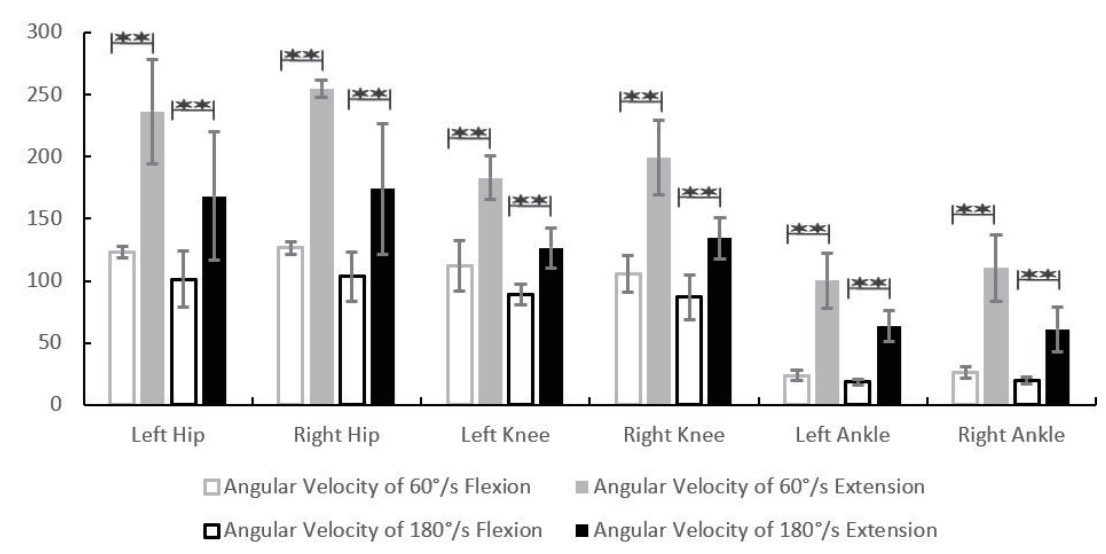

Fig. 4. Descriptive statistics for isokinetic peak torque (PT) of the lower extremities. **Significantly different between flexion and extension, $P<0.01$.

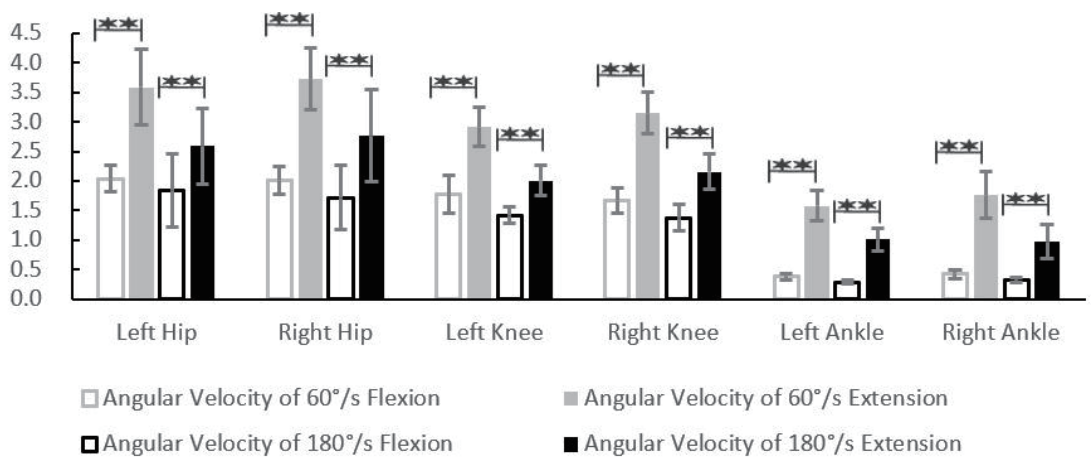

Fig. 5. Descriptive statistics for relative peak torque (PT/BW) of the lower extremities. ${ }^{* *}$ Significantly different between flexion and extension, $P<0.01$.

Table 3

Impact strengths of front and reverse shoulder tackles in different positions (mean $\pm \mathrm{SD}$ ).

\begin{tabular}{lccccccc}
\hline$N=18$ & \multicolumn{3}{c}{ Front shoulder tackle } & & \multicolumn{3}{c}{ Reverse shoulder tackle } \\
\cline { 1 - 2 } Position & High & Middle & Low & & High & Middle & Low \\
Impact strength (N/BW) & $1.06 \pm 0.09$ & $1.19 \pm 0.06$ & $1.18 \pm 0.07$ & & $1.00 \pm 0.14$ & $1.15 \pm 0.08$ & $1.10 \pm 0.11$ \\
Nonparametric test & 0.200 & 0.200 & 0.200 & & 0.200 & 0.089 & 0.053 \\
\hline
\end{tabular}

Table 4

Results of Pearson correlation analysis between isokinetic strengths of the lower-extremity joint extensors in slow movement and impact strength of shoulder tackle.

\begin{tabular}{lcc}
\hline \multirow{2}{*}{ Angular velocity: $60 \% \mathrm{~s}$} & \multicolumn{2}{c}{ Relative peak torque of extensors (R) } \\
\cline { 2 - 3 } & Left ankle & Right knee \\
\hline Impact strength of front shoulder tackle in high position & & $0.654^{*}$ \\
\hline Impact strength of front shoulder tackle in middle position & $0.593^{*}$ & $0.670^{* *}$ \\
\hline Impact strength of front shoulder tackle in low position & $0.609^{*}$ & $0.691^{* *}$ \\
\hline Impact strength of reverse shoulder tackle in high position & & $0.554^{*}$ \\
\hline Impact strength of reverse shoulder tackle in middle position & $0.548^{*}$ & $0.574^{*}$ \\
\hline Impact strength of reverse shoulder tackle in low position & &
\end{tabular}

*Significantly correlated at a $95 \%$ confidence interval (two-tailed test).

** Significantly correlated at a $99 \%$ confidence interval (two-tailed test). 


\section{Discussion}

\subsection{Difference in strength between lower-extremity flexors and extensors}

At the same angular velocity, the strengths of the extensors were significantly higher than those of the flexors, indicating that the function of the extensors was superior to that of the flexors under the same conditions. The reason for this difference was that the offense and defense in rugby sports, especially the shoulder tackle actions in defense, are mainly based on stretching and are supplemented by the buffer technique after touching the ground. The offense and defense in rugby sports are composed of multiple sudden stops and starts, as well as highly accelerated movements in changing directions. The shoulder tackle is the most frequently used tackling action in defense, accounting for $61 \%$ of all tackling actions, ${ }^{(17-19)}$ leading to different muscle strengths between the flexors and extensors.

\subsection{Impact of lower-extremity strength on shoulder tackle technique}

The lower-extremity movement of shoulder tackles involves asymmetrical stretching and a non-vertical jump. The movement torque of lower-extremity multiple joints can be expressed as net joint torque $=$ gravity torque + interactive dynamic torque + external contact torque + muscle torque. The net joint torque reflects the muscle strength. ${ }^{(16)}$ In the stretching stage, the extensors contract concentrically to generate a torque, which indicates that the muscle strength determines the vertical and horizontal momentum, and thus the body movement speed ${ }^{(6-8)}$ and ultimately the impact strength.

The relative peak torque of lower-extremity isokinetic strength at fixed angular velocities of 60 and $180 \%$ respectively reflect the low-speed and high-speed strengths of flexors and extensors. Muscle strength and the balanced development of the flexor and extensor strengths maintain the defensive effect of shoulder tackles as well as the balance and stability of shoulder tackle actions. In this study, isokinetic strength was correlated to the impact strength of shoulder tackles. Our results showed that the impact strength of shoulder tackles was positively correlated to the peak torque of the right knee and left ankle extensors. We also found that the impact of shoulder tackles was correlated to the extensor strengths of the front knee and back ankle joint of the dominant side. These correlations are consistent with the biomechanical characteristics of the special shoulder tackle technique and are the result of the shoulder tackle technique adapting to the features of the lower-extremity joints under exertion. Athletes are accustomed to adopting their right side for front shoulder tackle defense, and therefore the right knee and left ankle extensors could be easily activated at a suitable angle. On the one hand, through specialized strength training, especially through strength training of the knee and ankle extensors, the impact strength of shoulder tackles can be improved. On the other hand, because single training of the dominant-side shoulder tackle technique could cause an imbalance between the lower-extremity flexor and extensor strengths, specialized strength training is needed to evenly develop the strengths of the left-side and right-side flexors and extensors. 
Effective measures to improve shoulder tackle ability are to first develop the specialized strength of shoulder tackles in a balanced manner, especially the even development of the strengths of the left and right legs, as well as the flexors and extensors of the unilateral leg. Next, the left and right front shoulder tackle techniques need to be developed evenly. At the start of training, athletes should downplay the perspective of a dominant shoulder tackle, change their habit of using their dominant shoulder, and adopt left or right shoulder tackles based on different distances. The habitual adoption of tackle defense with the dominant shoulder could cause an imbalance of the strength between the left and right legs and increase the frequency of reverse shoulder tackle defense. During a reverse shoulder tackle with the opposite leg forward, the body can easily generate rotational torque, which affects the transmission of lowerextremity strength and the balance of the action. ${ }^{(20)}$ In short, a reverse shoulder tackle reduces the effectiveness of defense and easily leads to sports injuries.

\section{Conclusions}

We suggest that the lower-extremity isokinetic strengths of the front knee and back ankle in dominant-side tackles should be used as reference indexes to evaluate shoulder tackle ability. The balanced development of the lower-extremity strength and front and reverse shoulder tackle techniques can help athletes enhance the defensive capacity of shoulder tackles, promote technical learning, improve the stability of actions, and prevent sports injuries.

\section{Acknowledgments}

The authors thank all of the players, coaches, and conditioning staff associated with Shanghai University of Sport for their participation in this study and Sheng Ao-Li from Shanghai University of Sport of Kinesiology for her assistance with the data collection.

\section{References}

1 B. Y. Oudshoorn, H. F. Driscoll, M. Dunn, and D. James: J. Sports Sci. 36 (2018) 914. https://doi.org/10.1080/0 2640414.2017.1346273

2 T. J. Gabbett and S. Jaime: Plos One 11 (2016) e0163161. https://doi.org/10.1371/journal.pone.0163161.

3 M. J. Speranza, T. J. Gabbet, R. D. Johnston, and J. M. Sheppard: J. Strength Cond. Res. 29 (2015) 2071. https://doi.org/10.1519/JSC.0000000000000897

4 T. J. Gabbett, D. G. Jenkins, and B. Abernethy: J. Sports Sci. 29 (2011) 1655. https://doi.org/10.1080/02640414. 2011.610346

5 M. J. Speranza, T. J. Gabbett, R. D. Johnston, and J. M. Sheppard: Int. J. Sports Physiol. 10 (2015) 754. https:// doi.org/10.1123/ijspp.2015-0044

6 T. J. Gabbett, D. G. Jenkins, and B. Abernethy: J. Strength Cond. Res. 25 (2011) 72. https://doi.org/10.1519/ JSC.0b013e3181ff506f

7 T. J. Gabbett, D. G. Jenkins, and B. Abernethy: J. Strength Cond. Res. 24 (2010) 2989. https://doi.org/10.1519/ JSC.0b013e3181f00d22

8 T. J. Gabbett: J. Strength Cond. Res. 23 (2009) 540. https://doi.org/10.1519/jsc.0b013e31818efe8b

9 K. Kipp, J. Krzyszkowsk, and J. Heeneman: Sports Biomech. 1 (2019). https://doi.org/10.1080/14763141.2019.1 579854

10 P. Schons, R. G. D. Rosa, G. Fischer, and G. Fischerc: Sports Biomech. 18 (2019) 515. https://doi.org/10.1080/1 4763141.2018.1435712. 
11 J. Kirkpatrick and P. Comfort: J. Strength Cond. Res. 25 (2011) 1374. https://doi.org/10.1519/ JSC.0b013e3182804a6d

12 S. R. Brown, M. Brughelli, and P. C. Brughelli: Int. J. Sports Physiol. 9 (2014) 358. https://doi.org/10.1123/ IJSPP.2013-01

13 S. R. Brow, P. C. Brughelli, and L. Bridgeman: Int. J. Sports Physiol. 11 (2016) 500. https://doi.org/10.1123/ ijspp.2015-0241

14 I. J. Dobbs, C. M. Watkins, S. R. Barillas, and L. E. Brown: Isokinet Exerc. Sci. 25 (2017) 281. https://doi. org/10.3233/IES-171171

15 S. M. Anastasi and M. A. Hamzeh: Int. Conf. Net Pro. (IEEE, 2011) 198-203. https://content.iospress.com/ articles/isokinetics-and-exercisescience/ies00420

16 Z. Y. Lu, W. Li, and Q. Xie: Chi. Sport Sci. Tech. 52 (2016) 141. https://doi.org/10.16470/j.csst.201602021

17 N. Burger, M. I. Lambert, W. Viljoen, J. C. Brown, C. Readhead, and S. Hendricks: Br. J. Sports Med. 50 (2016) 932. https://doi.org/10.1136/bjsports-2015-095295

18 A. Ross, N. Gill, J. Cronin, and R. Malcata: Eur. J. Sport Sci. 15 (2015) 565. https://doi.org/10.1080/17461391.2 015.1029983

19 L. Vazl, M. V. Rooyen, and J. Sampaio: J. Sports Sci. Med. 9 (2010) 51. https://doi.org/10.1590/S180755092012000100011

20 T. Kawasaki, T. Yasumasa, H. Tanaka, H. Tanaka, and K. Kaneko: Am. J. Sports Med. 46 (2018) 2514. https:// doi.org/10.1177/0363546518781808 